\title{
Clinical observation on acupoint massage plus Vitalstim electrical stimulation for deglutition disorder after stroke
}

\section{穴位按摩结合 Vitalstim 电刺激治疗脑卒中后吞咽障碍临床观察}

\author{
Tian Li (田莉) ${ }^{1}$, Nie Shao-tong (聂绍通 $)^{2}$, Lou Tian-xiao (楼天晓 $)^{2}$, Chen Huan (陈欢) ${ }^{3}$, Yuan Guang-hui (袁光辉) ${ }^{3}$ \\ 1 Changsha Social Work College, Changsha 410000, China \\ 2 Hunan Traditional Chinese Medical College, Zhuzhou 412000, China \\ 3 Zhuzhou Central Hospital, Hunan Province, Zhuzhou 412000, China
}

\begin{abstract}
Objective: To observe the effect of acupoint massage plus Vitalstim electrical stimulation on deglutition function and surface electromyography (SEMG) of deglutition muscle groups.

Methods: A total of 60 patients with deglutition disorder after stroke were selected and divided into an electrical stimulation group, a massage group and an integrated group according to the random number table method, with 20 cases in each group. Patients in these three groups were given the same routine rehabilitation training for deglutition. In addition, patients in the electrical stimulation group were given extra Vitalstim electrical stimulation, patients in the massage group were given extra acupoint massage on the head, face and neck, and patients in the integrated group were given extra acupoint massage plus Vitalstim electrical stimulation. Fujishima Ichiro food intake level scale (FILS) was scored before and after treatment. The swallowing duration and maximal amplitude of masseter muscle in SEMG were evaluated before and after treatment.

Results: After treatment, the FILS score and the maximal amplitude of recruitment potential generated by muscular contraction of masseter muscle group in the three groups were higher than those before treatment (all $P<0.05)$, and the swallowing duration of masseter muscle group was shortened compared with that in the same group before treatment (all $P<0.05$ ). After treatment, the FILS score in the integrated group was higher than that in the electrical stimulation group and the massage group (both $P<0.05$ ). The swallowing duration of masseter muscle group measured by SEMG was lower than that in the electrical stimulation group and the massage group (both $P<0.05$ ), while the maximal amplitude was higher than that of the electrical stimulation group and the massage group $(P<0.05)$. After treatment, there were no significant differences in the FILS score, swallowing duration and maximal amplitude of masseter muscle group between the electrical stimulation group and the massage group (all $P>0.05$ ).

Conclusion: Both acupoint massage and electrical stimulation can improve the deglutition function in patients with deglutition disorder after stroke, and improve the coordination and flexibility of masseter muscle. The integration of the two is more effective.

Keywords: Acupoint Pressure Therapy; Electric Stimulation Therapy; Electromyography; Poststroke Syndrome; Pseudobulbar Palsy; Deglutition Disorders

【摘要】目的：观察穴位按摩结合Vitalstim电刺激对脑卒中后吞咽障碍患者吞咽功能及吞咽肌群表面肌电图的影 响。方法: 将60例脑卒中后吞咽障碍患者按照随机数字表法分为电刺激组、按摩组和联合组, 每组20例。三组均 采用相同的吞咽常规康复训练，电刺激组加用Vitalstim电刺激，按摩组加用头面颈项部穴位按摩，联合组加用 Vitalstim电刺激和穴位按摩。于治疗前后进行藤岛一郎吞咽困难分级量表(FILS)评分和表面肌电图中咬肌群的吞咽 时程、最大振幅评估。结果: 治疗后, 3组的FILS评分和咬肌群肌肉大力收缩时产生的募集电位最大振幅均较同组 治疗前提高(均 $P<0.05$ ), 咬肌群的吞咽时程均较同组治疗前缩短(均 $P<0.05$ )。治疗后, 联合组FILS评分高于电刺激 组和按摩组(均 $P<0.05$ ), 表面肌电图测量的咬肌群的吞咽时程低于电刺激组和按摩组(均 $P<0.05)$, 而最大振幅高 于电刺激组和按摩组 (均 $P<0.05)$ 。治疗后, 电刺激组和按摩组FILS评分、咬肌群的吞咽时程和最大振幅差异均无 统计学意义(均 $P>0.05)$ 。结论：穴位按摩及电刺激治疗均可改善脑卒中后吞咽障碍患者的吞咽功能，提高咬肌群 的协调性和灵活性，两种方法联合使用疗效更佳。
\end{abstract}

Author: Tian Li, M.M., associate chief physician, professor

Corresponding Author: Nie Shao-tong, bachelor, professor.

E-mail: 55617191@qq.com 
【关键词】点穴疗法; 电刺激疗法; 肌电描记术; 中风后遗症; 假性延髓麻廙; 吞咽障碍

【中图分类号】R246.6【文献标志码】A

The incidence of deglutition disorder after stroke is $30 \%-65 \%{ }^{[1-2]}$, the incidence of aspiration in patients with deglutition disorder after stroke is $51 \%-73 \%{ }^{[3-4]}$, and deglutition disorder causes $72 \%$ of hospitalized aspiration pneumonia ${ }^{[5]}$. Complications such as pneumonia will delay the recovery process of patients' swallowing function and even lead to death. Therefore, it is of great significance to take intervention as soon as possible. At present, the rehabilitation methods for deglutition disorder include oral sensory training, oral movement training, airway protection method, lowfrequency electrical stimulation, surface electromyographic biofeedback training, balloon dilatation, acupuncture treatment, application of ventilator swallowing and speaking valve ${ }^{[6]}$. In this study, acupoint massage and Vitalstim electrical stimulation were adopted to treat patients with deglutition disorder after stroke, and surface electromyography (SEMG) as an objective evaluation method, thus to provide reference for the selection of clinical treatment methods for patients with this disease.

\section{Clinical Materials}

\subsection{Diagnostic criteria}

\subsubsection{Diagnostic criteria for stroke}

The diagnostic criteria for stroke referred the Key Diagnostic Points for Cerebrovascular Diseases ${ }^{[7]}$.

1.1.2 Diagnostic criteria for deglutition disorder after stroke

The diagnostic criteria of Western medicine referred the definition of deglutition disorder after stroke in the Key Diagnostic Points for Cerebrovascular Diseases ${ }^{[7]}$ and Chinese Expert Consensus on Deglutition and Nutrition Management of Stroke Patients (2013 Edition) ${ }^{[8]}$. The diagnosis of stroke was confirmed by cranial CT or MRI examination, and deglutition disorder, choking while drinking water and slurred speech or voice disorder were demonstrated. The diagnostic criteria of traditional Chinese medicine (TCM) referred the Criteria of Diagnosis and Therapeutic Effects of Diseases and Syndromes in Traditional Chinese Medicine $^{[9]}$ and Standard for Diagnosis and Therapeutic Effect Evaluation of Stroke (Trial) ${ }^{[10]}$. The diagnosis was confirmed when the criteria for stroke disease in TCM were met, along with symptoms of deglutition disorder, choking while drinking water.

\subsection{Inclusion criteria}

Those who met the above diagnostic criteria, diagnosed with stroke by cranial CT or MRI examination, which was the first onset; screened as deglutition disorder by water swallowing test, incomplete loss of swallowing function, and no need to rely on nasal feeding; relatively stable vital signs, mini-mental state examination (MMSE) $\geq 21$ points, and could actively cooperate during rehabilitation training; aged between 41 and 70 years old; onset within 6 months; signed informed consent.

\subsection{Exclusion criteria}

Those with critical conditions; those with failure or bleeding tendency of important organs; those with severe cognitive impairment.

\subsection{Dropout criteria}

Those who were unwilling to continue the treatment; those with recurrent cerebrovascular diseases or aggravated condition during the study.

\subsection{Statistical methods}

The data was kept by a Microsoft Excel data sheet. The SPSS version 19.0 statistical software was adopted for data analysis. The measurement data in accordance with normal distribution were expressed as mean \pm standard deviation $(\bar{x} \pm s)$, and compared by analysis of variance. Chi-square test was used to compare the rate. Rank-sum test was used to compare ranked count data between groups. $P<0.05$ indicated statistical significance.

\subsection{General data}

A total of 60 inpatients with deglutition disorder after stroke who received treatment from the Department of Rehabilitation Medicine and Neurology of Zhuzhou Central Hospital, Hunan Province between February 2015 and September 2017 were recruited in the study. They were randomly divided into an electrical stimulation group, a massage group and an integrated group, with 20 cases in each group. No cases dropped out during treatment. There were no significant inter-group differences in gender, age and disease duration (all $P>0.05$ ), indicating that the three groups were comparable (Table 1).

\section{Therapeutic Methods}

Patients in the three groups received the same basic treatment, including drug treatment and routine rehabilitation training for swallowing.

The drug treatment followed the routine treatment of cerebrovascular disease in the neurology department stipulated in China Guidelines for Cerebrovascular Diseases Prevention and Treatment ${ }^{[11]}$, such as control of blood pressure, blood lipid, blood glucose, and anti-platelet aggregation. 
Table 1. Comparison of the general data among the three groups

\begin{tabular}{|c|c|c|c|c|c|c|c|}
\hline \multirow{2}{*}{ Group } & \multirow{2}{*}{$n$} & \multicolumn{2}{|c|}{ Gender (case) } & \multirow{2}{*}{$\begin{array}{l}\text { Average age } \\
(\bar{x} \pm s, \text { year })\end{array}$} & \multirow{2}{*}{$\begin{array}{l}\text { Average duration } \\
\qquad(\bar{x} \pm s \text {, day })\end{array}$} & \multicolumn{2}{|c|}{ Types of stroke (case) } \\
\hline & & Male & Female & & & Cerebral infarction & Cerebral hemorrhage \\
\hline Integrated & 20 & 13 & 7 & $61.4 \pm 9.1$ & $38.8 \pm 8.7$ & 10 & 10 \\
\hline Massage & 20 & 11 & 9 & $61.2 \pm 12.5$ & $36.4 \pm 9.5$ & 11 & 9 \\
\hline Electrical stimulation & 20 & 11 & 9 & $60.7 \pm 10.3$ & $37.3 \pm 9.3$ & 11 & 9 \\
\hline
\end{tabular}

Routine rehabilitation training for swallowing function included the following four categories.

Oral sensory stimulation: Asked the patient to take a sitting or semi-sitting position, and ice cotton stick or ice lemon cotton stick was used for ice or acid sensation stimulation. The stimulation sites were palatolingual arch, both sides of the soft palate, tongue root and posterior pharyngeal wall, and each site was stimulated 3-5 times.

Oral movement training: Passive, active and againstresistance movements with the mouth in closing and opening states, as well as extending the tongue in all directions. Each movement was repeated 3-5 times.

Chin tucking against resistance (CTAR) ${ }^{[12]}$ : Asked the patient to take a sitting position, and placed the small ball between the chin and the suprasternal fossa. Asked the patient to draw his chin downward and inward to squeeze the ball. Isometric contraction was maintained for $1 \mathrm{~min}$, followed by a 1-minute interval, and isotonic contraction was performed 30 times. This training was practiced for 3 times each morning, noon and evening.

Ingestion training: Swallowing angiography was adopted to determine the most suitable food consistency, properties and the amount of a mouthful. Chose an half lying or sitting position for eating, e.g. an half lying position with neck, slight forward flexion and a pillow under the hemiplegic shoulder. Selected food that was not easy to fall apart with even density. Made sure that each bite was swallowed before the next. Paid attention to the cleaning of oral secretions before and after eating.

\subsection{Integrated group}

\subsubsection{Acupoint massage}

Acupoints: Three acupoints on the face [Jiache (ST 6), Xiaguan (ST 7) and Chengjiang (CV 24)]; six acupoints on the neck [Lianquan (CV 23), Renying (ST 9), Tiantu (CV 22), Yamen (GV 15), Dazhui (GV 14) and Fengchi (GB 20)].

Methods: The location of acupoints referred the Nomenclature and Location of Acupuncture Points $\left(G B / T\right.$ 12346-2006) ${ }^{[13]}$. The patient took a supine position. Manipulations mainly included finger digital An-pressing, An-pressing, Rou-kneading and Tuipushing. The first step was to massage the three points on the face. The operator gently used the side of the middle finger and ring finger of both hands to push straight from Jiache (ST 6) to Xiaguan (ST 7) for 20-30 times. The operator Rou-kneaded and Anpressed the three points on the face with his thumb, two times of Rou-kneading and one An-pressing, each point for 10-20 times, until the patient felt sore and heavy. Then were the six points on the neck. The operator gently used the side of the two thumbs to push straight from Lianquan (CV 23) to Tiantu (CV 22) for 20-30 times, until the skin around the acupoints turned red. The operator digital An-pressed Lianquan (CV 23), Renying (ST 9) and Tiantu (CV 22) with his thumb for 10-20 times per acupoint, until the patient felt sore and heavy. The operator Rou-kneaded Lianquan (CV 23), Renying (ST 9) and Tiantu (CV 22) with his thumb for 10-20 times per acupoint. The operator Rou-kneaded Yamen (GV 15) to Dazhui (GV 14) for 10-20 times with the four fingers except the thumb. The operator Rou-kneaded Yamen (GV 15), Dazhui (GV 14) and Fengchi (GB 20) with his middle finger, each point for 10-20 times, until the patient felt sore and heavy. This treatment was given once a day for $6 \mathrm{~d}$ as a course, with a rest of $1 \mathrm{~d}$ between two courses and for 4 courses in total.

\subsubsection{Vitalstim electrical stimulation}

The skin was degreased first. The location and treatment mode of the surface electrode were selected based on the swallowing assessment results, the patient's tolerance as well as his disease condition. For patients with deglutition disorder of oral phase, the channel electrodes were placed at the body surface projection position of the buccal branch of facial nerve. For patients with deglutition disorder of pharyngeal phase, the channel electrodes were placed along the anterior midline, with one on the hyoid bone and the other on the cricoid cartilage. Treatment parameters: bidirectional square wave, wave width 600-700 $\mu \mathrm{s}$, frequency $30-80 \mathrm{~Hz}$, amplitude $0-25 \mathrm{~mA}$, dual-channel and dual-output electrodes were adopted. This treatment was given once a day for $30 \mathrm{~min}$ each time and $6 \mathrm{~d}$ as a course, with a rest of $1 \mathrm{~d}$ between two courses and for 4 courses in total.

\subsection{Massage group}

Patients in this group received the same acupoint massage treatment as in the integrated group, with the same acupoints, manipulations and treatment courses. 


\subsection{Electrical stimulation group}

Patients in this group received the same electrical stimulation treatment as in the integrated group, with the same stimulation sites, parameters and treatment courses.

\section{Observation of Clinical Efficacy}

\subsection{Observed items}

3.1.1 Score of Fujishima Ichiro food intake level scale (FILS)

Before treatment and after 4 treatment courses, FILS was scored ${ }^{[14]}$, between 1 and 10 grades from severe deglutition disorder to normal swallowing function, and the corresponding score was between 1 and 10 points. 1-3 points suggested severe disorder, in which patients were unable to eat orally; 4-6 points suggested moderate disorder, in which patients could eat orally, but supplementary nutrition was needed; 7-9 points suggested mild disorder, in which patients could take enough nutrition orally; 10 points suggested normal swallowing function.

\subsubsection{SEMG}

SEMG was adopted to quantitatively analyze the swallowing muscle function. The patient underwent SEMG examination before and after treatment. The examination environment should be quiet. Exposed the skin above the patient's neck, and used $75 \%$ alcohol to wipe the surface of electrodes to enhance their electrical conductivity. Took the left side as an example, stuck the recording electrodes under zygomatic arch, about $3 \mathrm{~cm}$ from earlobe (masseter muscle), and recording electrodes were $15-20 \mathrm{~mm}$ away from the reference electrodes. Asked the patient to swallow 15-20 mL purified water at one time as quickly as possible. If choking cough occurred, the measurement stopped and restarted after $20 \mathrm{~min}$. The recruitment pattern of the patient's swallowing process was intercepted, and the total duration (swallowing duration) and the maximal amplitude height of the recruitment potential generated during muscle contraction were measured. Both the left and right sides needed to be tested, and the average value of the two tests was taken as the evaluation result.

\subsection{Efficacy evaluation criteria}

Markedly effective: After treatment, swallowing function was significantly improved, and the FILS score was 9-10 points, or 5-7 points higher than that before treatment.

Effective: After treatment, swallowing function was improved, and the FILS score was 2-4 points higher than that before treatment.

Failure: After treatment, swallowing function did not change significantly, and the FILS score increased by less than 2 points.

\subsection{Results}

\subsubsection{Comparison of the efficacy}

The total effective rate in the integrated group was $95.0 \%$, versus $60.0 \%$ in the massage group, and $70.0 \%$ in the electrical stimulation group. The total effective rate in the integrated group was significantly higher than that in the other two groups $(P<0.05)$, and there was no significant difference between the massage group and the electrical stimulation group $(P>0.05)$. Please check Table 2 for details.

Table 2. Comparison of the efficacy among the three groups (case)

\begin{tabular}{lccccc}
\hline Group & $n$ & Markedly effective & Effective & Failure & Total effective rate (\%) \\
\hline Integrated & 20 & 13 & 6 & 1 & $95.0^{1)}$ \\
Massage & 20 & 6 & 9 & 5 & 60.0 \\
Electrical stimulation & 20 & 4 & 12 & 4 & 70.0
\end{tabular}

Note: Compared with the massage group and the electrical stimulation group, 1) $P<0.05$

\subsubsection{Comparison of the FILS score}

Before treatment, there was no significant intergroup difference in the FILS score $(P>0.05)$, indicating that the three groups were comparable. After 4 courses of treatment, the FILS scores in the three groups all increased significantly (all $P<0.05$ ). The score in the integrated group was higher than that in the massage group and the electrical stimulation group $(P<0.05)$. There was no significant difference between the massage group and the electrical stimulation group $(P>0.05)$. This suggested that all the three treatment methods could improve the FILS score, and the effect of the integrated treatment was better than that of the two treatment methods used alone. Please check Table 3 for details.

Table 3. Comparison of the FILS score among the three groups $(\bar{x} \pm s$, point $)$

\begin{tabular}{lccc} 
Group & $n$ & Before treatment & After treatment \\
\hline Integrated & 20 & $3.38 \pm 0.57$ & $7.69 \pm 0.64^{1)}$ \\
Massage & 20 & $3.32 \pm 0.58$ & $6.25 \pm 0.52^{1) 2}$ \\
Electrical stimulation & 20 & $3.35 \pm 0.55$ & $6.33 \pm 0.76^{1) 2}$ \\
\hline
\end{tabular}

Note: Compared with that before treatment in the same group, 1) $P<0.05$; compared with the integrated group after treatment, 2) $P<0.05$ 


\subsubsection{Comparison of the changes in the curves of} masseter muscle group in SEMG

Before treatment, there were no significant differences among the three groups in the swallowing duration and maximal amplitude of masseter muscle group (all $P>0.05$ ), indicating that the three groups were comparable. After 4 courses of treatment, the swallowing duration of masseter muscle group in the 3 groups was shortened, showing intra-group statistical significance (all $P<0.05$ ). The swallowing duration in the integrated group was shorter than that in the massage group and the electrical stimulation group (both $P<0.05)$. There was no significant difference between the massage group and the electrical stimulation group $(P>0.05)$. This suggested that all the three treatment methods could improve the coordination and flexibility of masseter muscle group, and shorten swallowing time.
And the effect of the integrated treatment was better than that of the two treatment methods used alone. After 4 courses of treatment, the maximal amplitudes of the recruitment potentials of masseter muscles in the three groups increased when the masseter muscles contracted vigorously, showing intra-group statistical significance (all $P<0.05$ ). The maximal amplitude in the integrated group was higher than that in the massage group and the electrical stimulation group (both $P<0.05)$, but there was no significant difference between the massage group and the electrical stimulation group $(P>0.05)$. This suggested that all the three treatment methods could enhance the masseter muscle and increase the discharge of muscle fibers, and the effect of the integrated treatment was better than that of the two treatment methods used alone. Please check Table 4 for details.

Table 4. Comparison of SEMG curves of masseter muscle group $(\bar{x} \pm s)$

\begin{tabular}{|c|c|c|c|c|c|}
\hline \multirow{2}{*}{ Group } & \multirow{2}{*}{$n$} & \multicolumn{2}{|c|}{ Swallowing duration (s) } & \multicolumn{2}{|c|}{ Maximal amplitude (mV) } \\
\hline & & Before treatment & After treatment & Before treatment & After treatment \\
\hline Integrated & 20 & $3.861 \pm 0.335$ & $3.172 \pm 0.327^{1)}$ & $0.297 \pm 0.051$ & $0.407 \pm 0.042^{1)}$ \\
\hline Massage & 20 & $3.876 \pm 0.283$ & $3.527 \pm 0.313^{1) 2)}$ & $0.305 \pm 0.044$ & $0.362 \pm 0.053^{1) 2)}$ \\
\hline Electrical stimulation & 20 & $3.866 \pm 0.356$ & $\left.3.530 \pm 0.347^{1) 2}\right)$ & $0.301 \pm 0.054$ & $0.332 \pm 0.021^{1) 2)}$ \\
\hline
\end{tabular}

Note: Compared with that before treatment in the same group, 1) $P<0.05$; compared with the integrated group after treatment, 2) $P<0.05$

\section{Discussion}

Acupoint stimulation is a common method to treat deglutition disorder after stroke nowadays. Qin L, et $a^{\left[{ }^{[15]}\right.}$ believe that deep needling at Lianquan (CV 23) and Yifeng (TE 17) can improve cervical nerve regulation, vascular nutrition and lymphatic circulation in patients with deglutition disorder after stroke. Zhang LZ, et al ${ }^{[16]}$ found that needling at Lianquan (CV 23) and Fengfu (GV 16) can affect the discharge of swallowing-related neurons in nucleus tractus solitarius, thus inducing swallowing movement. The research results of Yuan $Y$, et $a l^{[17]}$ suggested that stimulating deep sensation of the tongue and pharynx through deep needling at Tiantu (CV 22) can promote the recovery of swallowing muscle strength and central nervous system pathway. Some studies have also shown that repeated massage of lips, tongues and cheeks can effectively enhance the coordination of oral movements ${ }^{[18]}$; stimulation signals of massage are repeatedly transmitted to the cerebral cortex, thus improving swallowing function ${ }^{[19]}$.

The 9 acupoints selected in this study all function as regulating the swallowing function. Jiache (ST 6), Xiaguan (ST 7) and Chengjiang (CV 24) benefit the oral function. Renying (ST 9) is the crossing point of the Stomach Meridian and the Gallbladder Meridian. Renying (ST 9) and Lianquan (GV 23) are commonly used to treat deglutition disorder. Together with Tiantu
(CV 22), these three acupoints can unblock the meridians and benefit the pharynx ${ }^{[17,20]}$. Yamen (GV 15), the crossing point of the Governor Vessel and the Yang Link Vessel, can treat the loss of voice. Fengchi (GB 20) has the function of calming the liver and extinguishing the wind, as well as unblocking the collaterals and opening the orifices ${ }^{[21]}$. Dazhui (GV 14), the crossing point of the Governor Vessel and the yang meridians, can invigorate yang qi and regulate qi and blood.

Vitalstim electrical stimulation is one of the most popular methods to treat deglutition disorder in recent years. Some studies have shown that Vitalstim electrical stimulation can regulate muscle excitability, improve muscle strength of the swallowing muscle group and improve swallowing function by affecting motor fibers of medulla oblongata peripheral nerve ${ }^{[22]}$. Many research results have shown that Vitalstim electrical stimulation therapy is more effective than routine swallowing function rehabilitation training ${ }^{[23]}$.

The results of this study showed that acupoint stimulation, Vitalstim electrical stimulation and their integrated application can improve swallowing function in patients with deglutition disorder after stroke, and the effect of the integration of the two methods should be better than that of the two methods used alone.

SEMG, as a safe, simple and affordable evaluation method, is mostly adopted for early screening and evaluation of deglutition disorder ${ }^{[24]}$. It is attracting 
increasing attention at home and abroad as it can reflect the function of the swallowing muscle group. In recent years, more and more studies have taken SEMG as an objective indicator to evaluate the clinical efficacy of Vitalstim in treating deglutition disorder after stroke ${ }^{[25-26]}$. The results of SEMG examination in this study showed that after treatment, the swallowing durations of masseter muscle group in patients of the three groups were shortened, and the maximal amplitudes of muscle contraction were increased. This showed that acupoint stimulation, Vitalstim electrical stimulation and their integrated application can improve the flexibility and coordination of masseter muscle, enhance the muscle strength, and thus improve swallowing function. The effect of the integration of the two methods was better than that of the two methods used alone.

Acupoint massage is a non-invasive treatment, and it is safe and easy to operate. It can stimulate a larger area than acupuncture treatment, and the fear of patients for needling can be avoided, so that the method should be popularized and applied in clinical practice.

\section{Conflict of Interest}

The authors declare that there is no potential conflict of interest in this article.

\section{Acknowledgments}

This work was supported by Project of Hunan Province Administration of Traditional Chinese Medicine (湖南省 中医药管理局课题, No. 2015136).

\section{Statement of Informed Consent}

Informed consent was obtained from all individual participants.

Received: 20 December 2019/Accepted: 20 February 2020

\section{References}

[1] Martino R, Foley N, Bhogal S, Diamant N, Speechley M, Teasell R. Dysphagia after stroke: incidence, diagnosis, and pulmonary complications. Stroke, 2005, 36(12): 27562763.

[2] Roth EJ, Lovell L, Harvey RL, Heinemann AW, Semik P, Diaz S. Incidence of and risk factors for medical complications during stroke rehabilitation. Stroke, 2001, 32(2): 523-529.

[3] Sun WP, Ayiguli AS, Liu R, Sun W, Huang YN. Risk factors of aspiration in acute stroke patients. Zhongguo Kangfu Yixue Zazhi, 2010, 25(2): 131-134.

[4] Dou ZL. Assessment and Treatment of Dysphagia. 2nd Edition. Beijing: People's Medical Publishing House, 2017.

[5] Ning P, Yang JJ, Sun TY, Guo YF. Analysis of prevalence rate and risk factors for aspiration pneumonia in elderly inpatients. Zhonghua Laonianxue Zazhi, 2017, 36(4): 428432.
[6] China Expert Consensus Group on Rehabilitation Evaluation and Treatment of Deglutition Disorders. China expert consensus on rehabilitation evaluation and treatment of deglutition disorders (2017). Zhonghua Wuli Yixue Yu Kangfu Zazhi, 2017, 39(12): 881-892.

[7] Chinese Neuroscience Society, Chinese Neurosurgical Society. Key diagnostic points for cerebrovascular diseases. Zhonghua Shenjingke Zazhi, 1996, 29(6): 379-380.

[8] Chinese Expert Group on Deglutition Disorder and Nutrition Management in Stroke Patients. Chinese expert consensus on deglutition and nutrition management of stroke patients (2013 edition). Zhongguo Cuzhong Zazhi, 2013, 8(12): 973-983.

[9] State Administration of Traditional Chinese Medicine. Criteria of Diagnosis and Therapeutic Effects of Diseases and Syndromes in Traditional Chinese Medicine. Nanjing: Nanjing University Press, 1994: 119.

[10]Collaborative Group of Acute Encephalopathy of State Administration of Traditional Chinese Medicine. Standard for diagnosis and therapeutic effect evaluation of stroke (trial). Beijing Zhongyiyao Daxue Xuebao, 1996, 19(1): 55-56.

[11]Rao ML. China Guideline for Cerebrovascular Diseases Prevention and Treatment. Beijing: People's Medical Publishing House, 2007: 64.

[12]Yoon WL, Khoo JK, Rickard Liow SJ. Chin tuck against resistance (CTAR): new method for enhancing suprahyoid muscle activity using a Shaker-type exercise. Dysphagia, 2014, 29(2): 243-248.

[13] General Administration of Quality Supervision, Inspection and Quarantine of the People's Republic of China, Standardization Administration of the People's Republic of China. Nomenclature and Location of Acupuncture Points (GB/T 12346-2006). Beijing: Standards Press of China, 2006.

[14]Chang L, He PL, Zhou ZZ, Li YH. Efficacy observation of dysphagia after acute stroke treated with acupuncture and functional electric stimulation. Zhongguo Zhen Jiu, 2014, 34(8): 737-740.

[15]Qin L, Zhang XP, Yang XC, Cui CH, Shi J, Jia CS. Deep acupuncture of Lianquan (CV 23) and Yifeng (TE 17) in combination with conventional acupuncture of other acupoints is superior to swallowing rehabilitation training in improving poststroke dysphagia in apoplexy patients. Zhen Ci Yan Jiu, 2019, 44(2): 144-147.

[16]Zhang LZ, Xu NG, Li RL, Wang L. Clinical study of electroacupuncture with different frequencies at Lianquan (CV 23) and Fengfu (GV 16) for stroke dysphagia. Zhongguo Zhen Jiu, 2018, 38(2): 115-118.

[17] Yuan Y, Cai XH, Chen F, Chen DX, Gao Y, Liu ZZ, Ling $\mathrm{MX}, \mathrm{Xu}$ P. Clinical trials of acupuncture treatment of post-stroke dysphagia by deep acupuncture of Tiantu (CV 22) in combination with swallowing rehabilitation training. Zhen Ci Yan Jiu, 2019, 44(1): 47-50.

[18]Zheng H, Feng SM, Zhang JK, Ma BX. A clinical study on cerebral palsy with dysphagia by acupuncture combined with massage treatment. Zhongguo Kangfu Yixue Zazhi, 2014, 29(10): 918-922.

[19] Wang XQ, Bao CL. Clinical observation on acupoint massage combined with swallowing training in the 
treatment of stroke with dysphagia. Shanghai Zhongyiyao Daxue Xuebao, 2014, 28(5): 46-48.

[20]Li YN, Wang J, Zhou HF, Liu J, Zhang XF. Effects of different depths of acupuncture at Dicang (ST 4) and Jiache (ST 6) on the quality of life and facial nerve function of patients with peripheral facial paralysis. Zhongyi Zazhi, 2019, 60(2): 142-145.

[21] Han Q, Xu MS, Zhang YJ, Xu J, Ge LB. Effect of electroacupuncture at Fengchi (GB 20) on synaptophysin and growth associated protein- 43 in rats with ischemia/ reperfusion injury. Shanghai Zhenjiu Zazhi, 2019, 38(6): 674-680.

[22]Kushner DS, Peters K, Eroglu ST, Perless-Carroll M, Johnson-Greene D. Neuromuscular electrical stimulation efficacy in acute stroke feeding tube-dependent dysphagia during inpatient rehabilitation. Am J Phys Med Rehabil, 2013, 92(6): 486-495.

[23] Suntrup S, Marian T, Schröder BJ, Suttrup I, Muhle P, Oelenberg S, Hamacher C, Minnerup J, Warnecke T, Dziewas R. Electrical pharyngeal stimulation for dysphagia treatment in tracheotomized stroke patients: a randomized controlled trial. Intensive Care Med, 2015, 41(9): 16291637.

[24]Vaiman M, Eviatar E. Surface electromyography as a screening method for evaluation of dysphagia and odynophagia. Head Face Med, 2009, 5: 9.

[25] Li LJ, Li YM, Wu XH, Wang GH, Yi XJ, Zhao YC, Guo MJ, Pan MZ, Tang CL. The value of adding transcutaneous neuromuscular electrical stimulation (Vitalstim) to traditional therapy for post-stroke dysphagia: a randomized controlled trial. Eur J Phys Rehabil Med, 2015, 51(1): 71-78.

[26]Su WH, Yan WJ, Zhong MH, Li YM, Gao CF, Zhu QX. The effect of neuromuscular electrical stimulation on swallowing function after stroke. Zhonghua Wuli Yixue Yu Kangfu Zazhi, 2015, 37(3): 183-186.

Translator: Zhang Kai-wei (张凯维) 